\title{
Energy-aware caching and collaboration for green communication systems
}

\author{
Asif KABIR ${ }^{1}$, Syed Mushhad GILANI ${ }^{2}$, Gohar REHMANC ${ }^{3}$, S. SABAHAT H. ${ }^{4}$, \\ József POPP5*, Muhammad Arshad SHEHZAD HASSAN ${ }^{6}$ and Judit OLÁH ${ }^{7}$
}

\begin{abstract}
Authors' affiliations and addresses:
${ }^{1}$ Department of CS \& IT University of Kotli,11100,

Azad Jammu \& Kashmir, Pakistan

e-mail: asif.kabir@uokajk.edu.pk

${ }^{2}$ University Institute of Information Technology, PMASArid Agriculture University, 46000, Pakistan

e-mail: mushhad@uaar.edu.pk

${ }^{3}$ Women University of Azad Jammu and Kashmir, Bagh, Pakistan

e-mail: goharrehman25@yahoo.com

${ }^{4}$ School of Computer Science, Neijiang Normal

University, Suchuan, P.R China

e-mail: sabahatb2000@yahoo.com

${ }^{5}$ Hungarian University of Agriculture and Life Sciences, 2100 Gödöllö, Hungary; College of Business and Economics, University of Johannesburg, Johannesburg 2006, South Africa

e-mail:popp.jozsef@uni-mate.hu

${ }^{6}$ Department of Engineering Technology, The University of Faisalabad, Punjab 38000, Pakistan e-mail: arxhad@yahoo.com

${ }^{7}$ Faculty of Economics and Business, University of Debrecen, 4032 Debrecen, Hungary; College of Business and Economics, University of Johannesburg,

Johannesburg 2006, South Africa

e-mail: olah.judit@econ.unideb.hu

*Correspondence:

Hungarian University of Agriculture and Life Sciences, 2100 Gödöllö, Hungary; University of Johannesburg, Johannesburg 2006, South Africa e-mail: popp.jozsef@uni-mate.hu
\end{abstract}

\section{Acknowledgement:}

This research was supported by the János Bolyai Research Scholarship of the Hungarian Academy of Sciences and project no. 132805 has been implemented with the support provided from the National Research, Development and Innovation Fund of Hungary, financed under the K_19 funding scheme.

How to cite this article:

Kabir, K., Gilani, S. M., Rehmanc, G., Sabahat, S. H. Popp, J., Shehzad Hassan, M. A., and Oláh, J. (2021). Tax aspects of mining companies in V4 countries. Acta Montanistica Slovaca, Volume 26 (1), 47-59

DOI:

https://doi.org/10.46544/AMS.v26i1.04

\begin{abstract}
Social networks and mobile applications tend to enhance the need for high-quality content access. To meet the growing demand for data services in $5 \mathrm{G}$ cellular networks, it is important to develop effective content caching and distribution techniques, to reduce redundant data transmission and thereby improve network efficiency significantly. It is anticipated that energy harvesting and self-powered Small Base Stations' (SBS) are the rudimentary constituents of next-generation cellular networks. However, uncertainties in harvested energy are the primary reasons to opt for energy-efficient (EE) power control schemes to reduce SBS energy consumption and ensure the quality of services for users. Using edge collaborative caching, such EE design can also be achievable via the use of the content cache, decreasing the usage of capacity limited SBSs backhaul and reducing energy utilisation. Renewable energy (RE) harvesting technologies can be leveraged to manage the huge power demands of cellular networks. To reduce carbon footprint and improve energy efficiency, we tailored a more practical approach and propose green caching mechanisms for content distribution that utilise the content caching and renewable energy concept. Simulation results and analysis provide key insights that the proposed caching scheme brings a substantial improvement regarding content availability, cache storage capacity at the edge of cellular networks, enhances energy efficiency, and increases cache collaboration time up to $24 \%$. Furthermore, self-powered base stations and energy harvesting are an ultimate part of next-generation wireless networks, particularly in terms of optimum economic sustainability and green energy in developing countries for the evolution of mobile networks.
\end{abstract}

\section{Keywords}

Edge caching, renewable energy, energy efficiency, small cell cooperation, green communication, $5 \mathrm{G}$ 


\section{Introduction}

Due to the proliferation of smart devices and the explosion of traffic in the wireless ecosystem, cellular network operators face challenges of providing massive network capacity and achieving superior coverage while improving users' quality of experience. Small cell networks (SCN) are a cost-effective and energy-efficient network paradigm to tackle such challenges. In addition, according to the Small Cell Forum (Forum, 2015), from the perspective of cellular network operators around the globe, remote rural areas provide an excellent business opportunity for deploying small cells due to the increased coverage, provide services to potential customers and open new markets.

However, the deployment of small cells (SCs) in rural areas is a key challenge due to the backhaul availability and energy efficiency. Over the time in the telecommunication sector, the amount of energy consumption increases significantly. Many places, especially in emerging markets, do not have access or are limited to traditional grids, so the energy needs to be obtained from other renewable resources such as solar energy (Faludi and Gilbert, 2019). The amount of energy harvested varies over time, resulting in a potential power outage at the SBs, eventually decreasing the quality of services (QoS) for the users. Therefore, an energy-efficient (EE) transfer scheme must be implemented (Tvaronavičienè et al., 2018).

In addition, rural areas are often beyond the normal economic range of the MNO's existing backhaul infrastructure. This extensively enhances the backhaul cost, limiting the backhaul competence, making it complex to provide high-speed mobile broadband coverage. Due to the deployment of high-speed optical fibre backhaul, connecting the rural SBS with the core network is anticipated to be a very expensive process (Abdalla et al., 2016).

Moreover, to conquer these challenges, content caching at the network edge has appeared as one solution to offload backhaul traffic. However, there is variation in user requests and cache capacity at the edge of the network. So, employing the caching collaboration is similar to extending the cache size and makes the caching more capable. The cache collaboration ultimately reduces the power consumption and extends the edge cache size. Furthermore, when a user requests content to its serving base station, there is a need for an optimum cooperation scheme that saves energy in cooperation.

Numerous studies (Mehrabi et al., 2019, Usama and Erol-Kantarci, 2019 and Zahed et al., 2020) on the trend of edge caching energy and harvested $(\mathrm{EH})$ communications have been carried out in the past and different paradigms within shifted frameworks have been discussed in existing studies, such as green delivery (Ajaz Khan, 2019), which enables effective content distribution with energy harvesting based SCs. SCs provide content services based on energy status and content popularity. Similarly, authors Zhou et al. (2015) proposed that push content before the actual arrival of user demands which is known as proactively cache. Green Delivery is where HE-based small cells reduce grid energy utilisation and offer content services. In order to decrease the internet's carbon footprint, renewable energy-aware Energy Sharing, Trading in Multi- routing and caching for green communication, which minimises the grid energy usage, are presented in different studies (Oikonomakou et al., 2019, Mariyakhan et al., 2020). Studies on small cell distributed caches have also deduced that a cooperative caching scheme increases content distribution.

In green communications, network collaboration is seen as a way to save energy without increasing transmission power. This is achieved by alternately activating and deactivating the existing resources of the BS. Besides, reference by Han and Ansari (2016) emphasises maximising the throughput while meeting the constraints imposed by the renewable energy sources initial and storage of energy. Yao et al. (2015) studied a green mobile network with a cache that uses energy adaptation, sleep control schemes, and a tradeoff between energy and latency, which reduces power and network latency.

Similarly, by Mao et al. (2015), the comparison among the grid power consumption, EH-SBS and network performance was discussed. The authors investigate important characteristics, including network deployment, feasibility analysis, network operation problems and also presented a broad overview of energy harvesting SCN. Recently, it has been recommended by Kumar and Saad (2015) to enhance the EE by proposing a QoS online energy efficiency scheme that leverages the content of the SBSs' cache, which depends on totally renewable energy sources. Han and Ansari (2017) suggested a traffic load balancing scheme that considers network utilities.

This scheme compares different caching technologies and proposes a small cellular network that can be used for hybrid power caching. Edge caching approaches can potentially increase the energy efficiency of the core network by decreasing the burden on the backhaul and preventing multiple transmissions of similar content to different users.

Energy assessment models of end to end mobile networks have been studied by Yan and Chan (2019), and a service-specific end to end energy utilisation of each mobile network segment has been developed, involving the wireless access network, end-user devices, data centre, and core network.

Though edge caching is a comparatively new offloading method, it has, however, gained the attention of researchers. Over a certain time, the question is how to cooperatively design base station cooperation policies and caching techniques that reduce overall energy consumption. There is a growing consensus on the need to develop a more energy-efficient telecommunication system. Recent advancements in wireless network technologies have 
been accomplished to achieve energy efficiency in the context of rapidly rising energy prices and the explosive progress of mobile data traffic, and no tolerance of the QoS. Moreover, implement edge local caching at the SC in an energy-efficient way is still an open scientific challenge and needs further research efforts (Virglerova et al., 2020).

Reduction decreasing of energy consumption and edge cache delays have been analysed in some earlier work. The case of small cells by Virglerova et al. (2020) studied the interaction among energy and cache. At the same time, energy efficiency for small and dense cellular networks is an excellent initiative. Green energy enables networks to reduce their carbon footprint and directly affect climate change and the environment (Kath et al., 2020 and Chebli et al., 2020). However, modelling an optimal green energy situation for a cellular network is a challenging task. Network measurement analyses revealed that the energy consumption of SBSs is absolutely linked to traffic burdens (Barnett et al., 2018).

To enhance mobile networks, the capacity to satisfy the demands of a heterogeneous network is one of the important technologies. In HetNets low-power base stations are widely implemented to improve network efficiency. A cache at SBS utilises much less power than MBSs. To relieve on-grid power utilisation, the envelopment energy harvesting technology BSs can be powered by green energy. Liu and Yang (2016) studied a potential energy-efficient analysis of cache-enabled dense small cell networks, who revealed that cooperation caches at the edge of the networks for cellular network operators are significant in order to exploit the capacity and EE of SBSs. Similarly, by Yan et al. (2019), relay nodes cooperating with the user to cache contents to reduce the cooperative energy utilisation were examined. For improving system throughput in next-generation networks, dense small cell networks have been considered a promising solution.

Inspired by EH communications and the trends in edge caching, diverse paradigms of shifted frameworks have been intended, such as green delivery by Ik and Azeez (2020), enabling efficient content delivery with energy harvesting based SCs. Energy harvested SCs provide content services based on EH status and content popularity. Before the actual arrival of the user request, the small base stations proactively cache and push the content. Green delivery EH based SCs reduce energy consumption from the power grid and provide content services. To decrease the internet's carbon footprint and brown energy usage, presented renewable energy-aware routing and joint caching for green communication. SC distributed cache has been examined by Ao and Psounis (2017), who determined that a cooperative caching approach maximises content delivery. In a green radio communications network, cooperation is studied as a means of energy-saving without enhancing transmission power, which is accomplished by alternately switching on and off the existing resources from the BSs.

\section{Motivation and contribution}

We envisioned that the next-generation mobile networks would be furnished with renewable energy resources for the subsequent motives.

- $\quad$ RE effectively reduces the carbon footprint, and it is economical and environment-friendly, which impacts the deployment of cellular BSs in rural/remote areas.

- It solves the economic issues which mobile network operators are facing. A green objective has been added to the list of $5 \mathrm{G}$ goals to provide a cost-effective solution that reduces the overall cost of energy consumption, which constitutes around half of their operational expenditures (OPEX). Around 120TWh of electricity is consumed per year, costing 13 billion US\$ to mobile operators to serve the 5 billion connections per year (Göker and Ayar, 2020 and Kath et al., 2020).

- Due to improvements in renewable energy technology, the cost of RE, particularly solar, is probable to be fewer than that of non-renewable energy.

- RE has already been used in different power network equipment, such as Nokia Siemens base stations, Ericsson base stations and wireless routers.

- Information and communication technology not only provides room for green energy efficient systems but also helps other industrial sectors to improve their basic functionalities in the management of energyefficient systems. The ICT industry is also concerned about the potential benefits of renewable energy sources (RES) for a more ecological and sustainable design for future systems. Energy efficiency and renewable energies are the main pillars of sustainable development and environmental sustainability (Guermoui et al., 2020).

Thus, the cumulative consumption of these devices is high, which increases the trend towards edge caching and computing. Therefore, energy consumption will be higher in the future, and the use of renewable energy by Oláh et al. (2017) will be increased due to its tremendous benefits. Despite these facts and due to rapidly rising energy prices, environment-friendly condensers have already become a critical design factor in both mobile networks and low storage device prices (Bilan et al., 2019 and Suroso et al., 2020)

Meanwhile, the EE small cell networks are a significant concern. So, motivated by the above observation and discussion, we hereby propose an energy-efficient cache sharing scheme for energy harvested hybrids rural/ remote SBSs, where SBSs are endowed in a different way to the traditional cache techniques. So, we segregate this method 
into fixed and trending caching capabilities based on local and global content popularity distribution. Thus, the joint content cache will improve the backhaul latency and increase the efficiency of remote SBSs.

The rest of the paper is organised as follows. Section 3 defines our system model along with the formulation of the problem in detail, which also consists of wireless transmission, a user content cache model, and a power consumption model. In Section 4, we formulate our problem. In Section 5, we investigate the performance of our cache schemes. Finally, in Section 6, we present the conclusions of the paper.

\section{System model and problem formulation}

\section{System Model}

We analyse the energy efficiency problem for a future wireless cooperative network. Fig. 1 shows the proposed system model. Here, we have one $M B$ and a set of SBS $B=\left\{b_{1}, b_{2}, \ldots, b_{i}, b_{j}, \ldots, b_{n}\right\}$ which serves the users $U=\left\{u_{1}, u_{2}, \ldots, u_{k}\right\}$. Users are deployed randomly within a radius of SBSs and request content independently. Each SBS has a fixed storage size of content and has a library of $N$ local files denoted by $C=$ $\left\{f_{1}, f_{2}, \ldots, f_{n}\right\}$. Throughout in this article, we will use the terms content and file vice versa. Assume that user $u_{k}$ is serviced by the small base station $b_{i}$, the SBSs are connected with MBS, neighbour SBS via a backhaul and a virtual link, respectively. Each SBS is connected to the MBS and also its neighbour SBS $b_{n} \in M B$ and $b_{i}$ is connected to $b_{j}$. We assume that SBSs are aware of other SBSs' content information. The user requests content, and each user is served by one or multiple BSs depending upon the content location and transmission scheme. A user requests content $f_{x}$ from the associated base station $b_{i}$, and if the content is in the cache, it transmits the directory to the user. Otherwise, it takes from the neighbour's SBS, MBS, from the internet, or retrieving content from the internet generates traffic load in BS backhaul, depending upon the strategy.

We assume that SBSs are powered by the grid and green energy resources; it is also assumed that they have limited energy-saving capacity $\epsilon_{m}$. The energy harvesting condition is the same in each time slot $T=1 \ldots t$. There are total $K$ time slots, and the total time is therefore $K T$. Moreover, it is also considered that the data and channel information are shared with the proximate SBS. At each SBS, the scheduler performs resource allotment at the start of each time slot of a duration of $T$ seconds. Let the harvest energy $E_{k} \in\left[0, \epsilon_{m}\right]$ be the energy residual in the battery at the start of the kth time slot. Then the battery status is updated as

$$
E_{k+1}=\left(E_{k}-P_{k} T+A_{k}, \epsilon_{m}\right),
$$

Here, $A_{k}$ is a random variable representing the amount of energy harvested during the $T$ slot. The total transmission power can be represented by $P_{k}$ power consumption at the $k$-th time slot. If, as is likely, the userrequested content is not available in the local and adjacent SBS, the request must be forwarded to the MBS and the content provider, respectively.

Furthermore, there is $i$ content to be requested by the $k$-th user, so we use $S_{i}$ to signify the size of $i$-th content and $P_{k i}(t)$ to denote the projected amount of demand for content $i$ originating from the set at time $t$. Eu(t) represents the amount of consumed energy, with the symbol $r u \geq 0$ (joules/byte) used to denote the amount of energy utilised per unit to transmit the content to the user. The binary decision variable $x_{f}$ indicates whether a BS is used. Any file allowed to fetch

$$
x f=\left\{\begin{array}{l}
0: \text { if file } f \text { is not cached } \\
1: \text { if file } f \text { is cached }
\end{array}\right.
$$




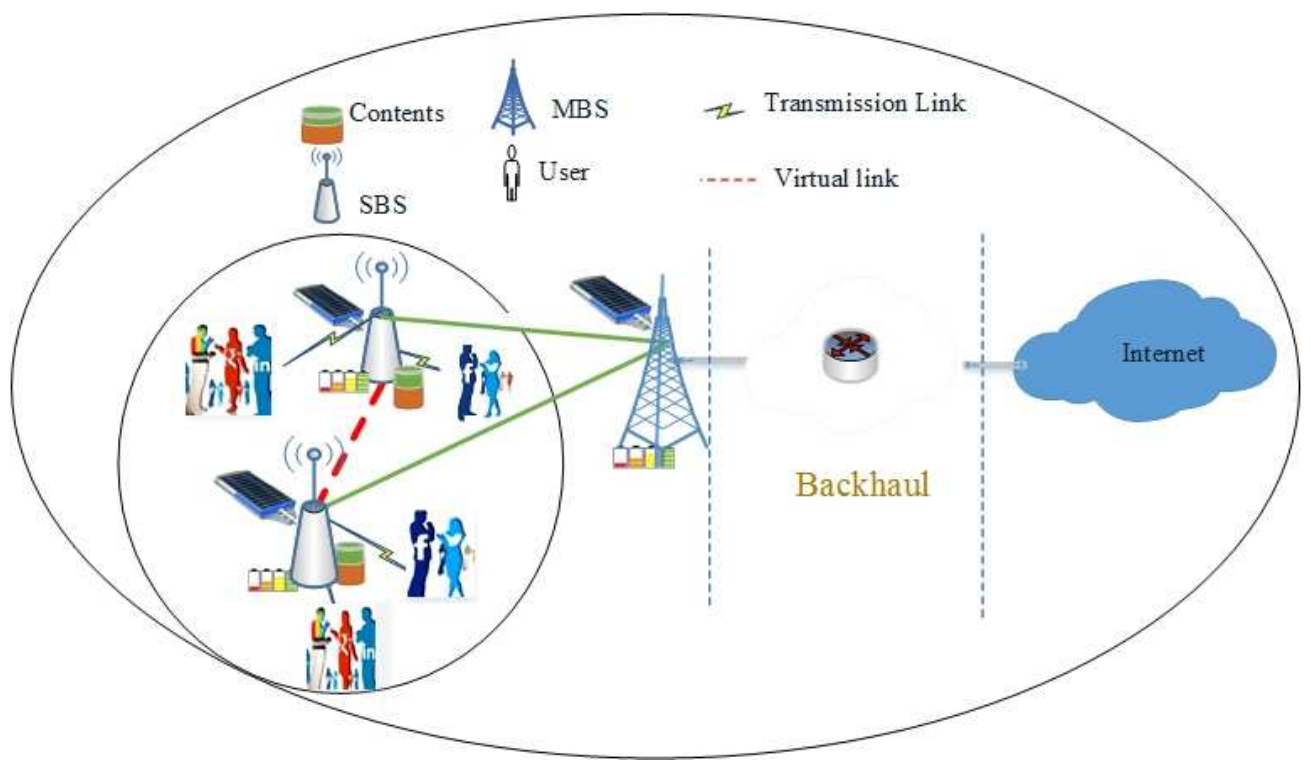

Fig. 1. System model

The user request content that consumes energy transmits power on the downlink for user $u_{k}$ during which period the kth slot can be represented by $P_{u, k}$. Similarly, transmission power consumed between the SBSs can be represented by $P_{b, k}$, fetching the content from the MBS, and the core network using the backhaul can be represented as $P_{M, k}$, and $P_{b k, k}$, respectively.

Here, the BSs cache capacity is distinct from conventional BSs that are not equipped with a cache. In the proposed scheme, the BSs are responsible for managing the entire wireless network, and network-related functions are employed in the BS, such as QoS management access control and connectivity.

\section{Content popularity distribution and estimation}

To reduce the use of backhaul to retrieve the required content from the core, we pretend that SBS caches the most popular content because of mostly the users' requests for popular content. As a result, popular content caching has the potential to cause lower latency and greater energy savings.

Moreover, data stored in remote content providers are based on the file popularity distribution and caching policy at each SBS. It is conceivable to determine the probability of the content $f$ requested by the user at a specific location. A user requested file in the local base station $p_{f}^{b_{u_{k}}}\left\{f \in\right.$ cache $b_{I}, f \in C_{i}$ user requested file is available at the local cache of an SBS $b_{i}$, and the cost to acquire $f$ from SBS $b_{i}$ is equal to zero. Hence, the probability that a user $u_{k}$ requests an uncached file is $f \notin C_{i}$, so, in this case $p_{f}=1-p_{f} u_{k} u_{i}$. There is another situation in which we have a collaborating cache scenario where the file may have an adjacent SBS; suppose it is in $b_{j}$. So, the probability, in this case, can be $p_{f_{u_{k}}}^{b_{j}}\left\{f \in\right.$ cache $b_{j}, f$ user requested and user request $\in U,\left\{b_{i}, b_{j}\right\} \in Z$. If the user requested file is unavailable in the neighbour, then it can be found in the cache of the macro base station; the probability distribution of the user-requested file in this case is $p_{f} t h e_{u_{k}}^{M B}=1-p_{f}^{b_{i}}-p_{f}^{b_{j}} u_{u_{k}}^{b_{j}}$. Finally, when the file is unavailable both locally and from an adjacent location, the file is retrieved from the content provider or CDN.

We estimated content popularity by Zipf's law. Typically, due to local user interest, the popularity of each content may fluctuate from place to place; this is captured through a localised request generation model, where the request pattern aggregate is different across various locations. Assume that $P_{i, f}$ denotes the popularity of content $f$ at $b_{i}$; considering the overall network, total popularity for each content is denoted as, $P_{f}=\sum_{i=1}^{n} P_{i, f}$, and we have

$$
P_{f}=\frac{k^{-r}}{\sum_{f=1}^{n} k^{-r}}
$$

Here, $k$ denotes the rank of popular content, $r \geq 0$ is a real constant $f$, and $k^{-r}$ is the skewness of popularity distribution. The higher $r$ value indicates that a fraction of the content is more popular than the rest of the catalogue and corresponds to a steeper distribution. In the vicinities of a caching entity, the popularity of the local content can be changed according to the different preferences of mobile users. Consequently, according to the diversity in local user content popularity, the proactive cache content placement should accommodate the maximise the 
number of cache hits. The files of the library are sorted in line with the descending order of popularity without a loss of generality, in which content "1" signifies the content with the highest downloading popularity.

$$
p_{f}^{b_{i}}+p_{f_{k}}^{b_{j}}+p_{u_{k}}^{b_{j}}{ }_{u_{k}}^{M B}+\left(1-p_{f_{u_{k}}}^{M B}\right)=1
$$

This means the multimedia content can always be delivered to the user. To fulfil the user's requirements, SBSs fetch the data from neighbouring cells, consuming energy. Here, among SBS and adjacent cells, ideal wireless backhaul links are considered to simplify the model derivation.

\section{Wireless transmission and the user content cache model}

We considered that a mobile user at location $\mathrm{x}$ is linked with the base station $\mathrm{b}_{\mathrm{i}}$. We also supposed that the number of users who requested interesting content and the traffic arriving at SBS $b_{i}$ follow the Poisson process with the parameter $\lambda$. Thus, it can be expressed as

$$
p(x f=f)=\frac{e^{-\lambda t}(\lambda t)^{f}}{f !},
$$

We pretend that users related to BS $b_{i}$ are evenly distributed in their coverage area, and the traffic arrival process is independent. Therefore, the traffic to users at any location is a Poisson process, and users at different locations may have different data rates, depending on the conditions of the channel. Though the BSs can use various access technologies as them backhaul, traffic distribution on the backhaul only provides a queuing system.

The wireless transmission model can be simplified as follows. If the user can download the interesting content from the connected SBS the transmission rate is set to be $C_{R}$ (Mbps). If the user can download the content from backhaul by the core network, this is represented by $C_{B}$ (Mbps). In cellular networks, the backhaul capacity of SBSs is generally limited. We further consider that $C_{B}<C_{R}$. In mobile networks, we emphasise on a multi-user orthogonal frequency division multiple access systems,

$$
\sum_{U} C_{B}<C_{R}
$$

where each channel of the system is orthogonal to the other. In other words, there is no interference between users. Therefore, users' wireless transmission speed depends on their available bandwidth and their signal-to-noise ratio.

\section{Content transmission model}

To achieve QoS in the proposed network scheme needs the consideration of green energy consumption, the performance of the cache system and BS capacity backhaul constraints. Traffic delivery latency in BSs and power consumption jointly help to increase the overall network performance. The transmission power between an SBS and a neighbour is represented as,

$$
P_{b, k}=€ P_{\circ}\left(\frac{d_{i}}{r_{0}}\right)^{\alpha},
$$

where $\epsilon$ is the coefficient variation under the normal mode, and $P_{o}$ is the value of SBS power utilisation. The distance among the SBS is denoted by $d_{i}$, and coverage is represented by $r_{o}$. Similarly, the energy utilisation used to transmit power between SBS and $u$-th user at time slot $k$ is

$$
P_{u, k}=\frac{\left({ }^{\frac{c_{S}}{b_{s}}}-1\right) d_{i}^{a_{S}}{ }_{i} \cdot \sigma^{2}}{\left\|h_{u, k}\right\|^{2}}
$$

where bandwidth is denoted by $b_{s}$ and $\boldsymbol{c}_{\boldsymbol{s}}$ is the transmission rate. We suppose that all users have the same bandwidth, the downlink channel among the user and SBS is denoted by $h_{u, k} d_{i}$, and the distance between the $u$-th user and the SBS and $\alpha$ is a path loss exponent. All channels are assumed to be i.i.d (independent and identically distributed) Rayleigh fading channels, with $\sigma^{2}$ representing the variance of the Gaussian noise.

When the user-requested content is not locally available, then user request must be sent to the core network, and the transmission of power between the MBS and the SBS and energy consumption is as follows, 


$$
P_{M B, k}=\frac{\left(2^{\frac{c_{m}}{b_{m}}}-1\right) d_{i}^{a_{m}} \cdot \sigma^{2}}{|| h_{b, k} \|^{2}},
$$

where the Rayleigh fading channel between the core network and SBS is denoted by $h_{b, k}$. The distance between the SBS and the MBS is represented by $d_{m}$. If the user's request content is not available in the local neighbour SBS cache or MBS, the request must be transmitted to the central network through the backhaul, and the SBS retransmits it on the downlink.

$$
P_{b k, k} P b k, k=\frac{\left(2^{\frac{c_{b k}}{b_{b k}}-1}\right) d_{i}^{a_{b k}} \cdot \sigma^{2}}{\left\|h_{b k, k}\right\|^{2}},
$$

Here, $d_{b k}$ is the distance between SBS and the content. Motivated by the realistic constraint, we assumed that utilising the backhaul connection consumes more power than retrieving information locally.

\section{Power consumption model}

In the network, we consider that the generating BS has its own RE system (solar). At the same time, the BS is also connected to the grid for power supply. Therefore, BS uses hybrid energy, namely brown and green energy. Fig. 2 shows the reference design of a hybrid energy power base station. When green energy is insufficient, BS consumes brown energy. MBS usually consumes more energy than SBS, so we assume that believe that the green energy system in MBS has greater energy production capacity than SBS. Based on the solar power potency the charge controller optimises the green power consumption, the power consumption of BSs and the process of energy drawn from the power grid. During a certain time period, the charge controller decides how much green power should be applied to power a BS. Generally, solar energy production is modeled on a one-hour timescale.

We represent $\epsilon_{m}$ as the quantity of green power used for powering the SBS $b_{i}$. If SBS $b_{i}$ power consumption is large, then $\epsilon_{m}$ the SBS utilises brown energy. Otherwise, we simply model the SBSs brown energy utilisation as zero. The power utilisation of SBS includes two parts: dynamic and static power utilisation. Static energy utilisation refers to the energy consumption of a BS that does not carry traffic loads. Dynamic power utilisation refers to the extra power utilisation caused by the traffic load on the BS. The power model can be assumed as follows.

The total power utilisation of BSs in the mobile network consists of operational power $P_{O C}$ and transmission power $\operatorname{cost} P_{t C}$. The total power cost can be represented as

$$
T_{P i}=\beta_{i} P_{o C}+P_{t c} \text {, }
$$

Here $\beta_{i}$ is the load power coefficient that signifies the relationship among BS $b_{i}$ dynamic power consumption and its traffic load; the BS $b_{i}$ brown power consumption is

$$
b_{i}^{b p}=\left(T_{P i}-\epsilon_{m}, 0\right) \text {, }
$$

Transmission occurs between the backhaul link of the BS, core network, as well as on wireless links between users and BSs. We consider the wireless part because wireless power consumption is usually dominant. Since the time considered is longer, we suppose that the demand for fast small-scale declines is average. Therefore, for the maximum coverage radius $(\mathrm{R})$ of the $\mathrm{BS}$, we focus on the impact of path loss to achieve the maximum transmission rate of all users covered by the BS with a BS greater than C. Due to transmission power, the maximum achievable transmission rate is:

$$
C=W_{u, b} \log _{2}\left(1+\frac{P_{t c} \beta R^{-\varepsilon}}{W_{u, b} \delta^{2}}\right),
$$

Here, $P_{t c}$ represent BS transmission power, $W$ represents the available bandwidth for each user, $\varepsilon$ is the path loss exponent, $\delta^{2}$ is the noise power, and $\beta$ is the path loss constant. We suppose that to satisfy a transmission delay requirement each transmission must meet a target rate, so the transmission power should satisfy the following.

$$
P_{t c}=\frac{\left(2^{C W_{u, b}{ }^{-1}}\right) W_{u, b} \delta^{2}}{R^{-\varepsilon}}
$$

\section{Problem formulation}

Our proposed scheme can be defined as reducing the transmission power by optimising the scheme for caching collaboration. To maximise energy efficiency, we need to consider various factors and practical constraints, such as local content accessibility, QoS requirements, backhaul capacity, channel state, interference level to maximise the EE, which satisfies QoS, and transmission power constraints.

Brown power is only used when green power is not sufficient $\left(\epsilon_{m}<b_{i}^{b p}\right)$. Given $\epsilon_{m}$, the maximum traffic load can be supported by green power in BS $b_{i}$. Green power is $0 \leq \epsilon_{m}<1$; when $\epsilon_{m} \leq 0$, it is hoped that the 
BS will bear additional traffic load to improve the use of green energy, thereby reducing the brown energy consumption of other BSs.

However, the balance between service power consumption and service congestion increases the waiting time for service transmission in the BS. In order to find the balance, we introduce a weighting factor for each BS, denoted as " $\omega$ ", which indicates the emphasis on time or energy costs.

$$
\omega * b_{i}=\epsilon_{m}^{k b_{i}}
$$

The BS latency weighting $k \geq 0$ is a system parameter that adjusts the value of the latency weight to save energy and reduce the latency for network traffic transmission. Let $E_{i, j}$ be the energy cost of the SBS with which user $i$ accesses the content from the BS $b_{j}$. We can calculate $E_{i, j}$ as,

$$
E_{i, j}=\left\{\begin{array}{l}
0 \text { if } d_{i, j}=0 \\
\text { else } \left.d_{i, b i}+d_{i, b j}+d_{i, m b}+d_{i,\left(1-d_{i, j}\right.}\right)
\end{array},\right.
$$

where $d_{i, j}$ is the mean distance of accessing the contents, and 0 is the content in the local cache, and there is no latency in accessing the content and energy cost. So, the energy consumption of accessing the content is $E=$ $\sum_{i=1}^{n} \sum_{u}^{k} p f_{i, k} E_{i j}$. To minimise the energy utilisation of a wireless collaboration caching network by finding the optimal content in the SBS and the local cache, we thus have the following optimisation problem

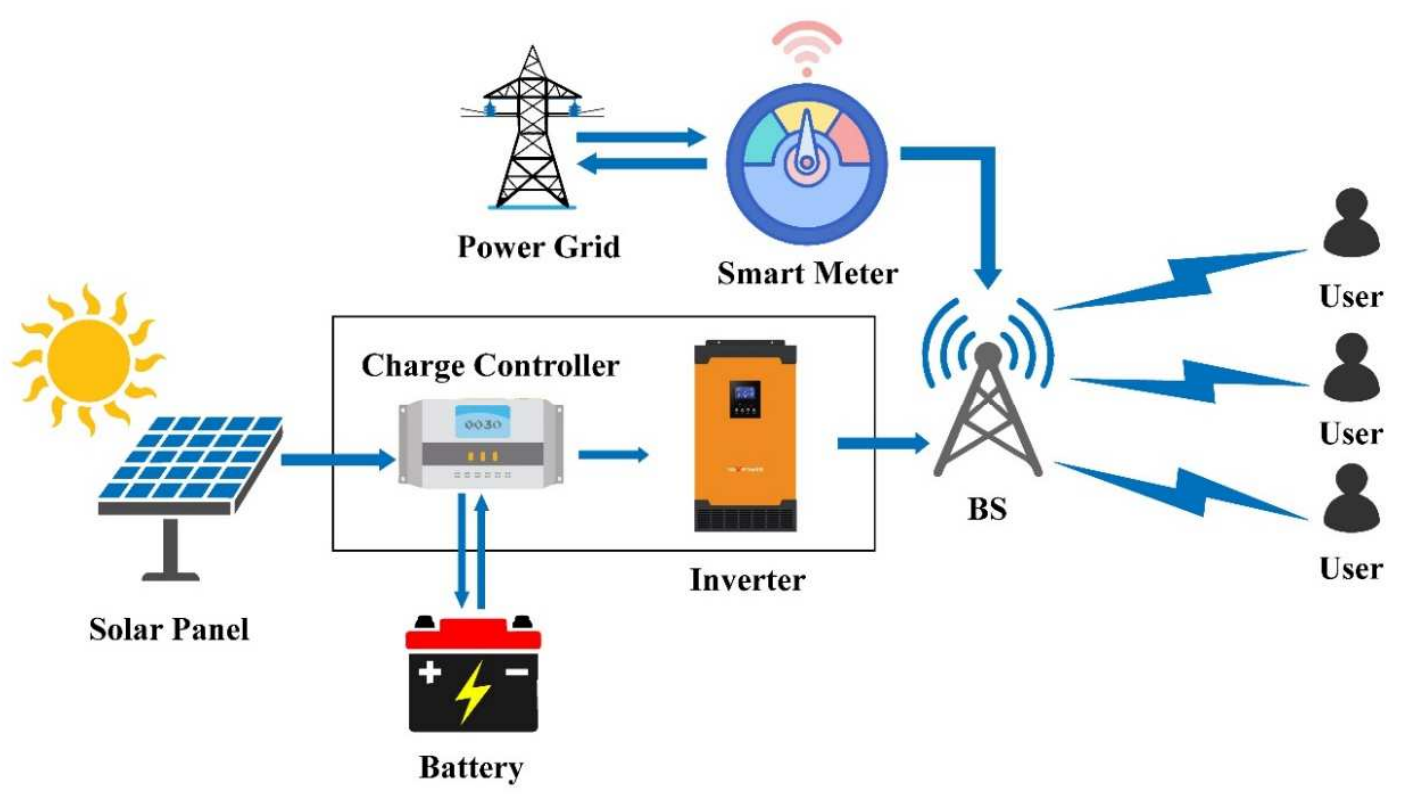

Fig. 2. A hybrid energy power base station.

$$
\begin{aligned}
& \min E_{i, j}, \\
& \text { Subject to } \forall c \in C, 0 \leq P_{i, f} \leq, \\
& \forall u_{k} \in b_{i}, \\
& \sum f_{s} \leq c, \\
& \qquad E_{k} \in\left[0, \epsilon_{m}\right], \\
& \qquad E_{k} \in\left[0, \epsilon_{m}\right],
\end{aligned}
$$

In this article, we use a joint optimisation method to delay costs while optimising energy consumption in the BSs, which points to the transmission power overlay network design of a small cellular network with the capability 
of content caching. The purpose of this heuristic approach is to decrease energy utilisation when accessing cached content in small cell base stations.

\section{Results and discussion}

In this article, we argue that cooperative caching and transmission costs will decrease the total power utilisation in cellular networks. We consider a cellular network where users are deployed randomly, and BSs are a spatial Poisson point process (SPPP) distributed in a circle of radius $R=100 \mathrm{~m}$ with a density of $\lambda=2 \times$ $10^{-4} / \mathrm{m}^{2}$. The popularity distribution of content follows Zipf's law with default exponents $r=0.8$. The separation between core and SBS is set to $500 \mathrm{~m}$. Battery capacity $\epsilon_{m}$ is $2 j$ and the maximum transmission power of an SBS Pk is $0.8 \mathrm{~W}$. It is assumed that the energy arrival process follows a Poisson distribution. The duration of the time interval is set to $\mathrm{T}=1 \mathrm{~ms}$. The files have the same size, and the cache size $\mathrm{C}=100 \mathrm{~GB}$ represents the number of cached files, and users can send requests at each time interval. The caching content stored in the SBS should be different according to the local SBS content popularity, where the value of the path loss exponents $\mathrm{a}_{s}, \mathrm{a}_{m}$ and the noise variance $\sigma^{2}$ is set to $90 \mathrm{dBm}$, and $\mathrm{a}_{b k}$ are set to be 3 . The motivation of this work is to understand the impact of caching and energy harvesting at an SBS. For simplification, we assume an equal bandwidth $W=100 \mathrm{kHz}$, for each user and backhaul. The power required to relay one item of content is set to $0.5 W$ per 1500 bytes of packets.

Fig. 3 explores the effect of the Zipf parameter. The average backhaul delay from SBS, MBS, and internet is represented by delay1, delay2, and delay3, respectively. The slope of the curves shows the content request in terms of the average latency in hybrid power green base stations. The proposed scheme significantly reduces the average delay and improves the cache at the edge of networks. The shape of the parameter of file popularity varies from a value of 0.5 to 3 . It can be clearly observed that the average delay decreases as the Zipf parameter rise.

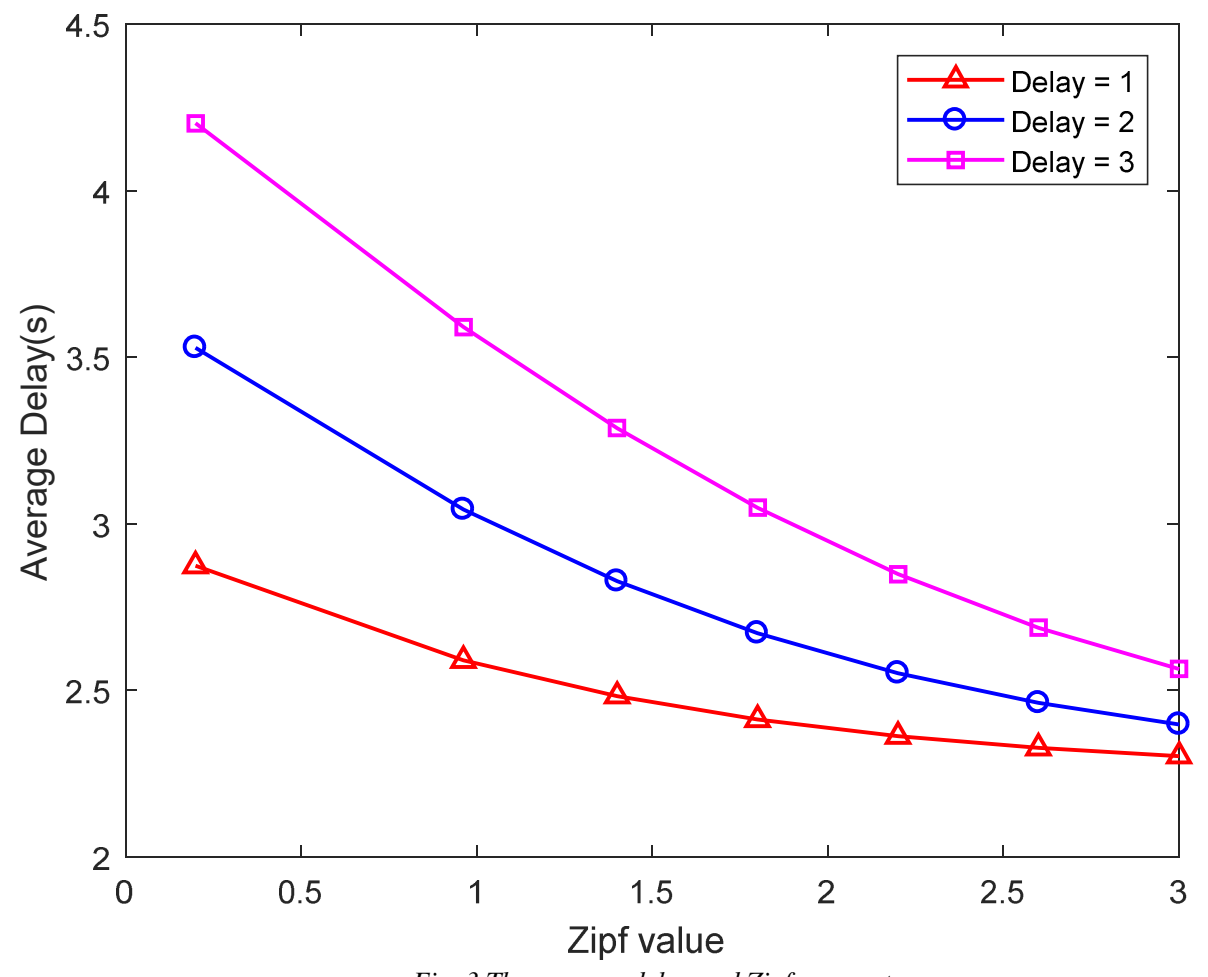

Fig. 3.The average delay and Zipf parameter.

In addition, under this network scenario, it can be noticed that, on average, as the Zipf parameter increases, the backhaul delay decreases. This is because when popularity distribution gets steeper and as the Zipf parameter is increased, a small number of contents are more popular, which improves the EE and cache effectiveness. Consequently, more content can be served directly from local caches of SBS, and they cannot travel through the backhaul, which minimises the effect of backhaul latency and energy. 


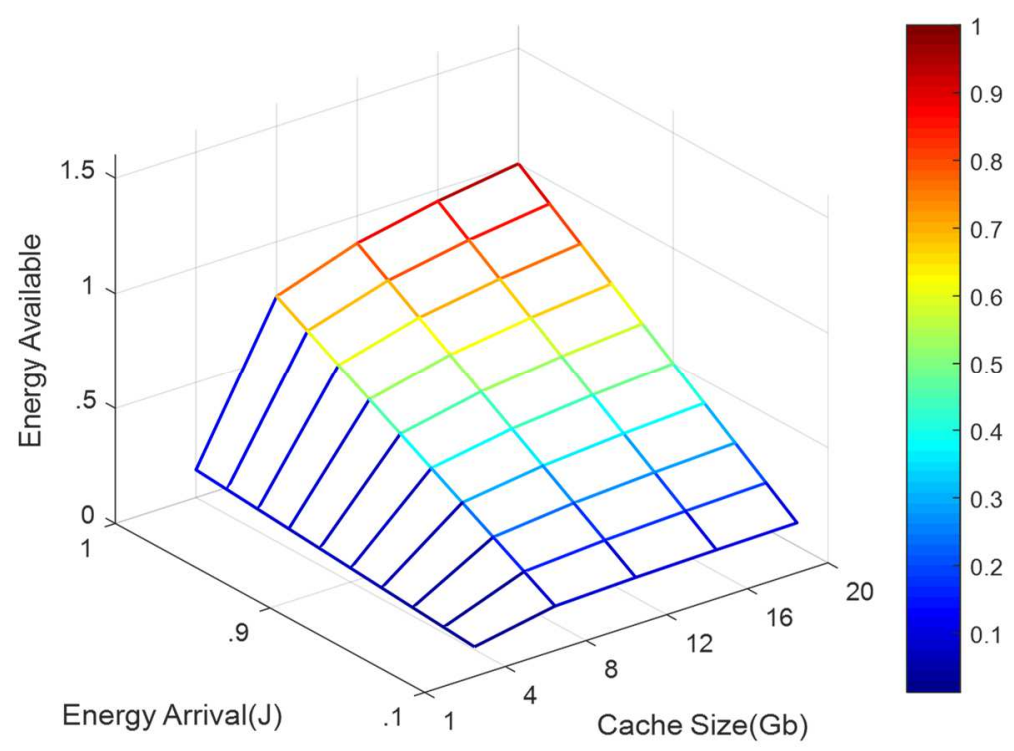

Fig. 4. The tradeoff between energy harvesting and cache size.

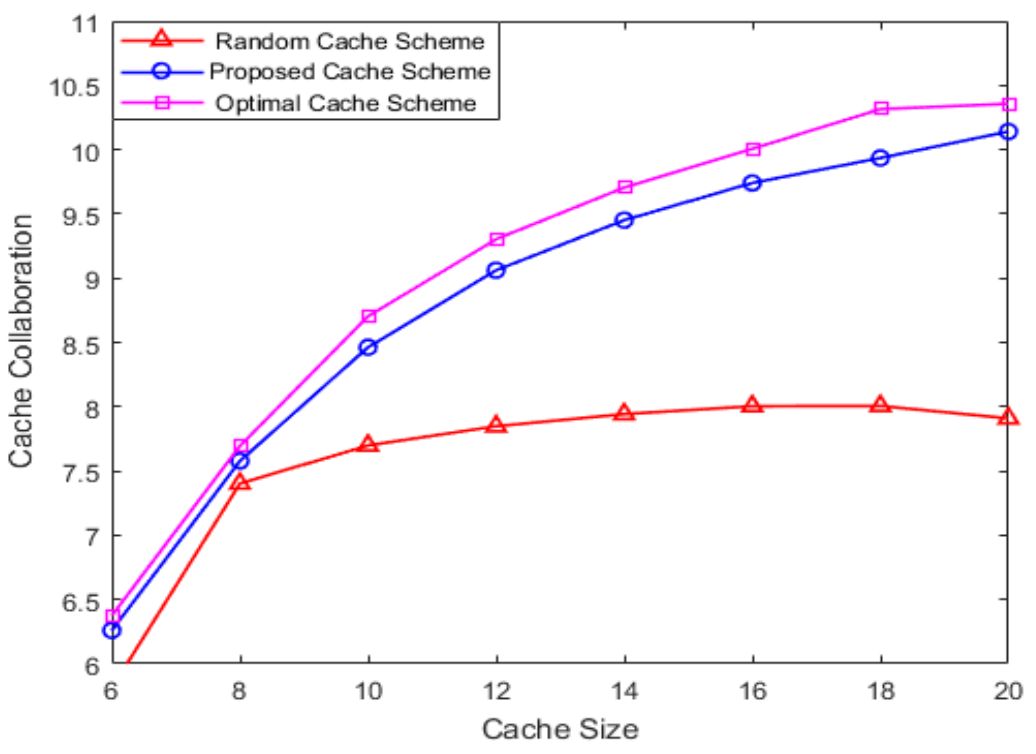

Fig. 5. Cache cooperation time vs cache size.

The tradeoff between the energy harvesting capacity and cache size to achieve energy harvesting performance is shown in Fig. 4. In order to achieve the desired green energy efficiency, we showed the energy available, the energy harvest per arrival and the impact of cache size at SBS. We concluded that by increasing the cache storage space from 1 to 20, or the harvested energy per arrival, the energy availability of the system goes up from 0 to 1.5 . MNO can make a better decision on the particular implication of this result about the required energy harvesting size and cache size of the device that achieves the required performance.

It is also noted that increasing the harvested energy has a more pronounced effect than increasing the cache size. Fig. 5 demonstrates the time of cache cooperation effects on the cache size in SBS. Within the energy harvesting rate, we observed that the cache collaboration time increases with the size of storage space, and SBS also increase in the proposed method. Due to the energy limitation, in the random scheme, the cache cooperation time fluctuates about 8 with a cache size of 8 to 20. For the proposed scheme and optimal scheme, the growth becomes slighter as the cache size becomes larger, i.e. the stage 6 to 14 has a great impact as compared to stage 16 to 20 on content matching. In addition, the energy harvested affects the size of the cache, and the proposed scheme results in a $24 \%$ increase in cache collaboration time compared to the random scheme cache. Fig. 6 . shows the influence of energy harvesting on cache collaboration time; we observed the cache cooperation as a function of energy harvesting rates. In the random cache, energy harvesting increases linearly with cache collaboration. This 
is due to the fact that energy is a crucial factor in cache coordination within an SBS. The caching cooperation times become almost stable at 1.3 to 2 for proposed and optimal.

Because the energy is abundant and the caching times cannot increase with energy arrival. The energy almost completely satisfies the caching collaboration cost when the energy harvesting rate is equal to 1.3. So, the arrival of more energy leads to better cooperation. Also, there is limited battery capacity, so energy harvesting cannot be stored fully.

Fig. 7 shows the distinct number of users versus energy cooperation. As we can see in all schemes, as the number of users increases, so does the average energy utilisation because more users will generate more demand, which will increase energy consumption costs. So, it can be observed that the scheme proposed in the current study consumes the least energy. From Fig. 7, we also observed that the slope of the curve is almost the same as the fixed cooperation time. We also observed that the proposed scheme saves significant energy as compared to random caching. Thus cache coordination between SBSs is a very applicable and energy-saving technique.

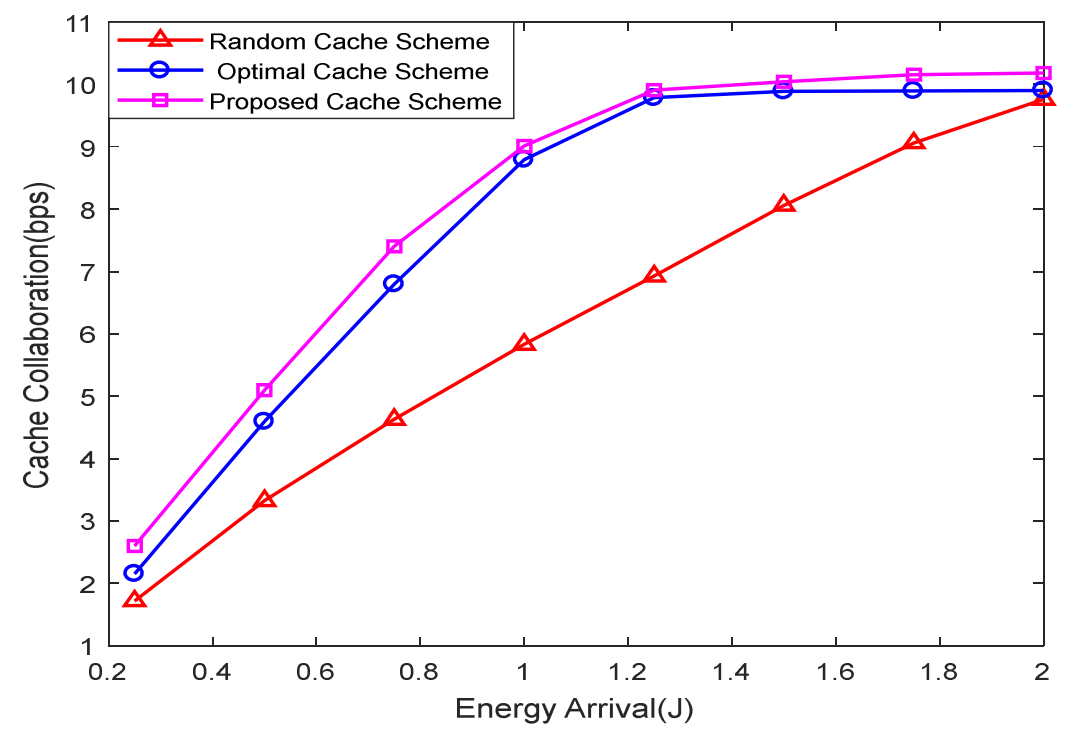

Fig.1 Energy harvesting on cache collaboration

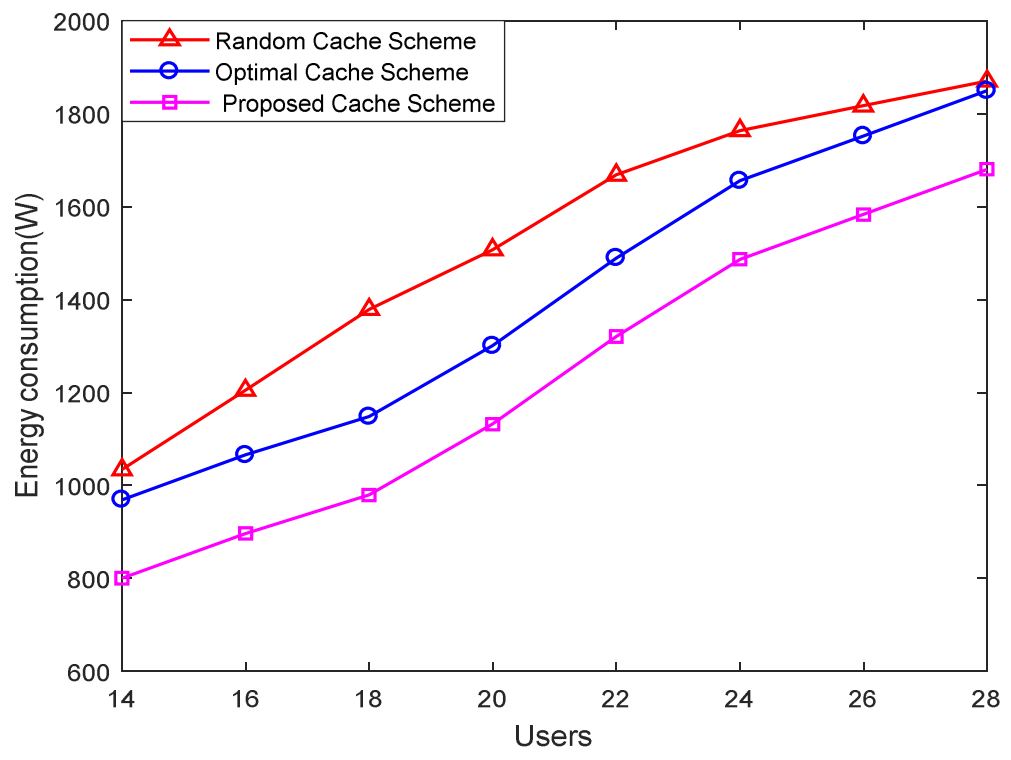

Fig. 7. Average energy consumption versus the number of users.

\section{Conclusion}

In this article, we have developed a wireless cooperative caching scheme and an energy-efficient power control scheme to minimise the energy utilisation for an energy harvesting SBS equipped with local storage and wireless backhaul. The key objective of this article is to evaluate the edge caching system in the context of cellular 
networks and maximise energy efficiency and determine its potency as a viable method for handling the exponential growth in wireless data traffic envisioned for the next-generation wireless networks. Inspired by the emerging energy harvesting communication in next-generation networks and the trend of edge content caching, we proposed a green delivery framework where harvesting is based on small cells sharing the content and minimising the transmission energy consumption. In addition, content popularity, energy arrival, and limited storage capacity are also considered. The numerical results demonstrated that the proposed EH cooperative edge caching scheme has a significant performance.

Moreover, an end to end latency is an important metric that is also related, and the tradeoff between these parameters gives further momentum to MNO for the sustainable development of cellular networks. We also consider energy arrival and limited storage capacity. Renewable energy not only provides economic benefits but also protects the environment from harmful greenhouse gas emissions.

Our method can be used as an important tool for congested backhaul and for extracting maximum benefit in network performance. The findings presented here have wider applicability than the scope of this paper. Finally, in the future, we would like to evaluate the results in more detail to explore the more features of edge cache with RE for next-generation EE and echo friendly cellular networks.

Also, there are several directions that can be further extended like RE, EE, reduce global warming are now the wish and attainment of all the important stakeholders that are linked with technology. Similarly, online cache and data in cellular edge network for analysis and design of context information big data is a valuable resource. Information and communication technology not only provides room for green energy efficient systems but also helps other industrial sectors to improve their basic functionalities in the management of energy-efficient systems. The ICT industry is also concerned about the potential benefits of renewable energy sources for a more ecological and sustainable design for future systems. Energy efficiency and renewable energies are the main pillars of sustainable development and environmental sustainability. As the main contribution, both aspects in which ICT makes our world ecologically sustainable are analysed more comprehensively, and the use of renewable energies in cellular communication is emphasised.

\section{References}

Abdalla, H. B., Lin, J., Li, G., \& Gilani, S. M. M. (2016). NoSQL: Confidential on Data Security and Data Management by Using a Mobile Application. International Journal of Information and Electronics Engineering, 6(2), 84-88. https://doi.org/10.18178/ijiee.2016.6.2.600

Ajaz Khan, K., Çera, G., \& Netek, V. (2019). Perception of the Selected Business Environment Aspects by Service Firms. Journal of Tourism and Services, 10(19), 111-127. https://doi.org/10.29036/jots.v10i19.115

Ao, W. C., \& Psounis, K. (2017). Fast Content Delivery via Distributed Caching and Small Cell Cooperation. In IEEE Transactions on Mobile Computing. https://doi.org/10.1109/TMC.2017.2750143

Barnett, T., Jain, S., Andra, U., \& Khurana, T. (2018). Cisco visual networking index (vni) complete forecast update, 2017-2022. Americas/EMEAR Cisco Knowledge Network (CKN) Presentation.

Bilan, Y., Streimikiene, D., Vasylieva, T., Lyulyov, O., Pimonenko, T., \& Pavlyk, A. (2019). Linking between Renewable Energy, CO 2 Emissions, and Economic Growth: Challenges for Candidates and Potential Candidates for the EU Membership. 1-16. https://doi.org/10.3390/su11061528

Chebli, A., Chabou Othmani, M., \& Ben Said, F. (2020). Market Segmentation in Urban Tourism: Exploring the Influence of Personal Factors on Tourists' Perception. Journal of Tourism and Services, 11(20), 74-108. https://doi.org/10.29036/jots.v11i20.144

Faludi, J., \& Gilbert, C. (2019). Best practices for teaching green invention: Interviews on design, engineering, and business education. Journal of Cleaner Production, 234, 1246-1261. https://doi.org/10.1016/j.jclepro.2019.06.246

Forum,S.C.(2015).Small

Forum. http://scf.io/en/documents/047_Extending_rural_and_remote_coverage_using_small_cells.php/Extending

Göker, G., \& Ayar, İ. (2020). Intermediary Role Of Nostalgia Tendency In The Effect Of Electronic Word Of Mouth Communication On Tourists' Destination Visit Intentions. Journal of Tourism and Services, 11(20), 44-59. https://doi.org/10.29036/jots.v11i20.140

Guermoui, M., Melgani, F., Gairaa, K., \& Mekhalfi, M. L. (2020). A comprehensive review of hybrid models for solar radiation forecasting. Journal of Cleaner Production, 258. https://doi.org/10.1016/j.jclepro.2020.120357

Han, T., \& Ansari, N. (2016). Provisioning Green Energy for Base Stations in Heterogeneous Networks. IEEE Transactions on Vehicular Technology, 65(7), 5439-5448. https://doi.org/10.1109/TVT.2015.2466101

Han, T., \& Ansari, N. (2017). Network Utility Aware Traffic Load Balancing in Backhaul-Constrained CacheEnabled Small Cell Networks with Hybrid Power Supplies. IEEE Transactions on Mobile Computing, 16(10), 2819-2832. https://doi.org/10.1109/TMC.2017.2652464 
Ik, M., \& Azeez, A. A. (2020). Organisational Green Behavioural Change: The Role of Change Management. International Journal of Entrepreneurial Knowledge, 8(1), 34-48. https://doi.org/10.37335/ijek.v8i2.98

Oláh, J., Lengyel, P., Balogh, P., Harangi-Rákos M., \& Popp, J. (2017). The role of biofuels in food commodity prices volatility and land use. Journal of Competitiveness, 9(4), 81-93. https://doi.org/ 10.7441/joc.2017.04.06

Kabir, A., Rehman, G., Gilani, S. M., Kitindi, E. J., Jaffri, Z. U. A., \& Abbasi, K. M. (2019). The role of caching in next generation cellular networks: A survey and research outlook. Transactions on Emerging Telecommunications Technologies, June, 1-25. https://doi.org/10.1002/ett.3702

Kath, C., Nitka, W., Serafin, T., Weron, T., Zaleski, P., \& Weron, R. (2020). Balancing generation from renewable energy sources: Profitability of an energy trader. Energies, 13(1), 1-15. https://doi.org/10.3390/en13010205

Kumar, A., \& Saad, W. (2015). On the tradeoff between energy harvesting and caching in wireless networks. 2015 IEEE International Conference on Communication Workshop, ICCW 2015, 1976-1981. https://doi.org/10.1109/ICCW.2015.7247470

Liu, D., \& Yang, C. (2016). Energy Efficiency of Downlink Networks with Caching at Base Stations. IEEE Journal on Selected Areas in Communications, 34(4), 907-922. https://doi.org/10.1109/JSAC.2016.2549398

Mao, Y., Luo, Y., Zhang, J., \& Letaief, K. B. (2015). Energy harvesting small cell networks: Feasibility, deployment, and operation. IEEE Communications Magazine, 53(6), 94-101. https://doi.org/10.1109/MCOM.2015.7120023

Mariyakhan, K., Mohamued, E. A., Asif Khan, M., Popp, J., \& Oláh, J. (2020). Does the level of absorptive capacity matter for carbon intensity? Evidence from the USA and China. Energies, 13(2), 407, 1-19. https://doi.org/10.3390/en13020407, https://www.mdpi.com/1996-1073/13/2/407

Mehrabi, A., Siekkinen, M., \& Yla-Jaaski, A. (2019). Energy-Aware QoE and Backhaul Traffic Optimization in Green Edge Adaptive Mobile Video Streaming. IEEE Transactions on Green Communications and Networking, 3(3), 828-839. https://doi.org/10.1109/TGCN.2019.2918847

Oikonomakou, M., Member, S., Antonopoulos, A., Member, S., Alonso, L., Member, S., Verikoukis, C., \& Member, S. (2019). Energy Sharing and Trading in Multi-Operator Heterogeneous Network Deployments. IEEE Transactions on Vehicular Technology, PP(XX), 1. https://doi.org/10.1109/TVT.2019.2902925

Suroso, A., Rafinda, A., \& Gal, T. (2020). The Evaluation Of Entrepreneur Incubation Program At Higher Education. International Journal of Entrepreneurial Knowledge, 8(2), 14-26. https://doi.org/10.37335/ijek.v8i2.113

Škare, M., Tomic, D., \& Stjepanovic, S. (2020). Energy Consumption and Green GDP in Europe: A Panel Cointegration Analysis 2008-2016. Acta Montanistica Slovaca, 25(1).

Tvaronavičienė, M., Prakapienė, D., Garškaitė-Milvydienė, K., Prakapas, R., \& Nawrot, Ł. (2018). Energy efficiency in the long run in the selected European countries. Economics and Sociology, 11(1), 245-254. https://doi.org/10.14254/2071-789X.2018/11-1/16

Usama, M., \& Erol-Kantarci, M. (2019). A survey on recent trends and open issues in energy efficiency of 5G. Sensors (Switzerland), 19(14). https://doi.org/10.3390/s19143126

Virglerova, Z., Conte, F., Amoah, J., \& Massaro, M. R. (2020). The Perception Of Legal Risk And Its Impact On The Business of SMES. International Journal of Entrepreneurial Knowledge, 8(2), 1-13. https://doi.org/10.37335/ijek.v8i2.115

Yan, M., Chan, C. A., Li, W., Lei, L., Gygax, A. F., \& I, C.-L. (2019). Assessing the Energy Consumption of Proactive Mobile Edge Caching in Wireless Networks. IEEE Access, 7, 104394-104404. https://doi.org/10.1109/access.2019.2931449

Yang, C., Chen, Z., Yao, Y., Xia, B., \& Liu, H. (2014). Energy efficiency in wireless cooperative caching networks. Communications (ICC), 2014 IEEE International Conference On, 4975-4980. https://doi.org/10.1109/ICC.2014.6884109

Yao, H., Fang, C., Qiu, C., Zhao, C., \& Liu, Y. (2015). A novel energy efficiency algorithm in green mobile networks with cache. Eurasip Journal on Wireless Communications and Networking, 2015(1). https://doi.org/10.1186/s13638-015-0373-7

Zahed, M. I. A., Ahmad, I., Habibi, D., Phung, Q. V., \& Mowla, M. M. (2020). Proactive content caching using surplus renewable energy: A win-win solution for both network service and energy providers. Future Generation Computer Systems, 105, 210-221. https://doi.org/10.1016/j.future.2019.11.041

Zhou, S., Gong, J., Zhou, Z., Chen, W., \& Niu, Z. (2015). GreenDelivery: Proactive content caching and push with energy-harvesting-based small cells. IEEE Communications Magazine, 53(4), 142-149. https://doi.org/10.1109/MCOM.2015.7081087 